\title{
GEM: International Journal on Geomathematics
}

\author{
Willi Freeden
}

Published online: 9 July 2010

(C) Springer-Verlag 2010

The special importance of mathematics as an interdisciplinary science in technology, economy, and commerce has been increasingly acknowledged within the last few years. This process did not remain without effects on mathematics itself. New mathematical disciplines, such as scientific computing, financial and business mathematics, industrial mathematics, biomathematics, and also mathematics concerned with geoscientific problems, i.e., geomathematics, have complemented the traditional disciplines. Today, computers and measurement technology have resulted in an explosive propagation of mathematics in almost every area of society. Mathematics as an interdisciplinary science can be found in almost every area of our lives. Mathematics is closely interacting with almost every other science ("mathematization of sciences"). The use of computers allows for the handling of complicated models for real data sets. Modeling, computation, and visualization yield reliable simulations of processes and products. Mathematics is the "raw material" for the models and the essence of each computer simulation. As the key technology, it translates images of the real world to models of the virtual world, and vice versa.

On our planet Earth, there has been a large number of far reaching changes during the last few years, e.g., species extinction, climate change, formation of deserts, anomalous ocean currents, disturbed structure of the atmosphere, transition of the dipole structure of the magnetic field to a quadrupole structure, etc. These changes have been accelerated dramatically. The main reasons for most of these phenomena are the unrestricted growth in the industrial societies (population and consumption, especially of resources, territory, and energy) and severe poverty in the developing and newly industrialized countries. The dangerous aspect is that extreme changes have taken place within a very short time; for many phenomena there has been no compa-

\footnotetext{
W. Freeden $(\varangle)$

University of Kaiserslautern, Geomathematics Group, P.O. Box 3049, 67653 Kaiserslautern, Germany

e-mail: freeden@mathematik.uni-kl.de
} 
rable development in the dynamics of the system Earth in the past. Changes brought about by man are much faster than changes due to natural fluctuations. The appalling résumé is that the geoscientific problems collected over the decades must almost all be solved simultaneously now. Interdisciplinary solutions including intensive mathematical research are urgently required as answers to an increasingly complex world. In consequence, mathematics is absolutely essential for a sustainable development in the future.

The intent of the journal GEM is to deal with the qualitative and quantitative properties of the current or possible structures of the system Earth. GEM provides concepts of scientific research concerning the system Earth, and geomathematics is simultaneously the force behind it. The research object, i.e., the system Earth, consists of a number of elements which represent individual systems themselves. The complexity of the entire system Earth is determined by interacting physical, biological, and chemical processes transforming and transporting energy, material, and information. It is characterized by natural, social, and economic processes influencing one another. All these aspects require a type of mathematics which must be more than a mere collection of theories and numerical methods. Geomathematics has to be concerned with nothing more than the organization of the complexity of the system Earth. Descriptive thinking is required in order to clarify abstract complex situations. Correct simplifications of complicated interactions, exact thinking and formulations are needed, numerical realizations (modeling and simulation) must be given, etc. All in all, geomathematics represents the key science of the complex system Earth. Wherever there are data and observations to be processed, e.g., the diverse scalar, vectorial, and tensorial clusters of satellite data, we need geomathematics. Whenever modeling and simulation come into play for the system Earth, geomathematics is decisive.

The specific task of geomathematics is to build a bridge between mathematical theory and geophyiscal as well as geotechnical applications. The special attraction is based on the vivid communication between mathematicians more interested in model development, theoretical foundation, and the approximate as well as computational solution of problems, and engineers and physicists more familiar with measuring technology, methods of data analysis, implementation of routines, and software applications. There is a very wide range of modern geosciences on which geomathematics is focused (see Fig. 1), not least because of the continuously increasing observational diversity. Simultaneously, the mathematical "toolbox" is becoming larger. A special feature is that geomathematics primarily deals with those regions of the Earth which are only insufficiently or not at all accessible for direct measurements (even by remote sensing methods). Inverse methods are absolutely essential for the mathematical evaluation in these cases. Mostly, a physical field is measured in the vicinity of the Earth's surface, and is then continued downward or upward by mathematical methods until one reaches the interesting depths or heights.

The scientific challenge does not only consist of increasing the leading role of mathematics within the current "scientific consortium Earth". The significance of the subject Earth must also be acknowledged (again) within mathematics itself, so that mathematicians will become more enthusiastic about it. Up to now, it has become usual and good practice in application-oriented mathematical departments and research institutions to present applications in technology, economy, finances, and even biology and 


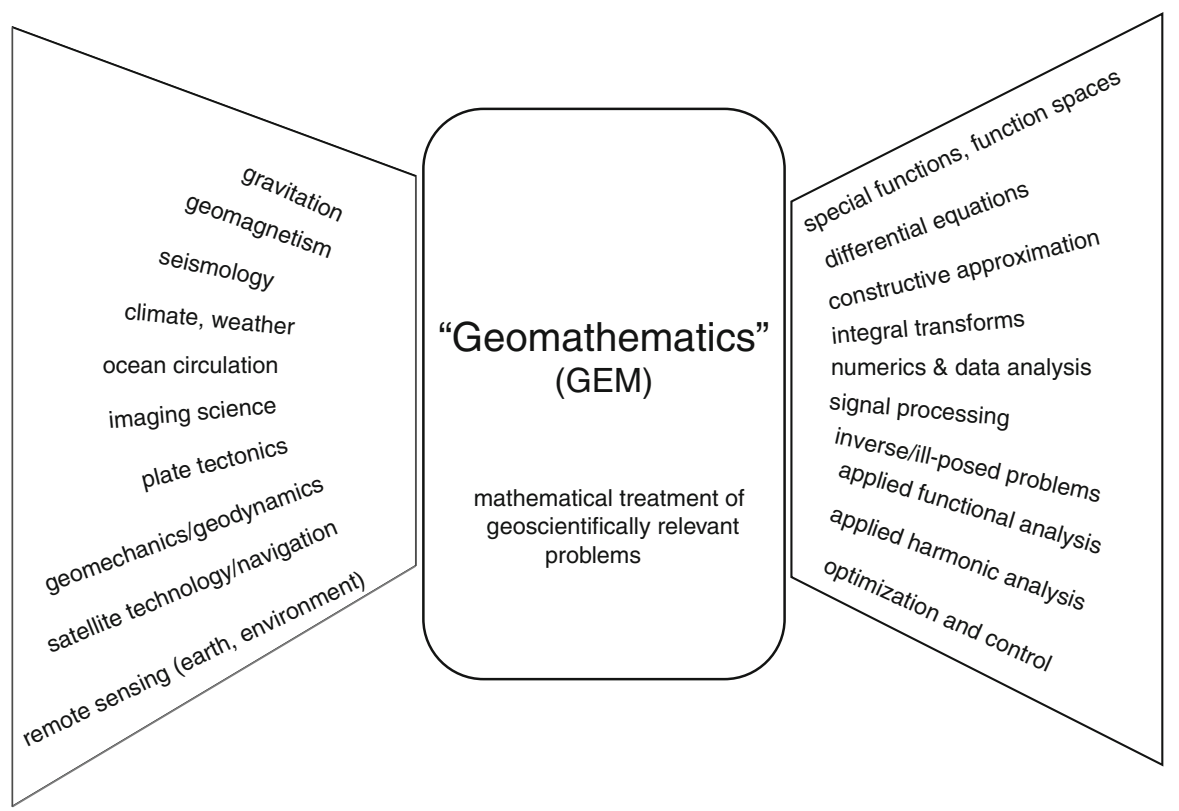

Fig. 1 Geomathematics, its range of fields, and its tools

medicine as being very career-enhancing for young scientists. Geomathematics can be integrated smoothly into this phalanx with current subjects like exploration, geothermal research, navigation, and so on. Mathematics should be the leading science for the solution of these complex and economically very interesting problems, instead of fulfilling mere service functions. Mathematics should not hide behind the other geosciences! Now is the time to turn expressively towards georelevant applications. The Earth as a complex, however, limited system (with its global problems concerning climate, environment, resources, and population) needs new scientific manifestations. For all these reasons, I dare to make the prognosis that GEM will become an essential platform for accepting and establishing a general responsibility of mathematics in our society.

GEM is intended to publish peer-reviewed mathematical papers that deal with

(i) modeling and simulation of the system Earth (geosphere, cryosphere, hydrosphere, atmosphere, biosphere, anthroposphere)

(ii) analytic, algebraic, operator-theoretic, and statistical methods

(iii) computational and numerical analysis methods necessary for the mathematical treatment of geoscientifically relevant problems.

As GEM Editor-in-Chief, I am honored to feature a collection of papers from world renowned scientists in the inaugural issue. I believe that the five papers demonstrate the high-quality, excellence, and relevance that can be expected from the journal. My goal is that GEM will emerge as an active and dynamic forum for the dissemination of creative and innovative ideas and concepts that will help to understand the Earth as our living environment. Moreover, I wish you will enjoy the journal and make it a great success in geomathematically driven and oriented science. 